\title{
Understanding the Role of Endoscopic Ultrasonography in Early Gastric Cancer
}

\author{
Chan Hyuk Park* and Sang Kil Lee ${ }^{\dagger}$
}

${ }^{*}$ Department of Internal Medicine, Hanyang University Guri Hospital, Hanyang University College of Medicine, Guri, and ${ }^{\dagger}$ Division of Gastroenterology, Department of Internal Medicine, Severance Hospital, Institute of Gastroenterology, Yonsei University College of Medicine, Seoul, Korea

See "Therapeutic Decision-Making Using Endoscopic Ultrasonography in Endoscopic Treatment of Early Gastric Cancer" by Jong Yeul Lee, et al. on page 42, Vol. 10. No. 1, 2016

Endoscopic submucosal dissection (ESD) has been widely used to treat early gastric cancer (EGC) with expanded indications. $^{1,2}$ The expanded indications for ESD proposed by Gotoda ${ }^{3}$ consist of four categories based on a combination of multiple tumor characteristics including tumor differentiation, depth of tumor invasion, tumor size, presence of ulcers, and presence of lymphovascular invasion. However, predicting whether a tumor fulfills the indication criteria by using endoscopy with biopsy alone is difficult. ${ }^{1}$ When a tumor resected through ESD does not meet the ESD indication criteria, subsequent surgery should be performed because of the possibility of lymph node metastasis. However, some patients with EGC undergo unnecessary surgery even when the lesion meets the ESD indication criteria.

To solve this problem, endoscopic ultrasonography (EUS) has been performed to increase the accuracy of predicting the depth of tumor invasion. Meta-analyses have shown the high sensitivity and specificity of EUS in predicting the T stage of gastric cancer. ${ }^{4,5}$ However, the ability of EUS to distinguish mucosal cancer from submucosal cancer remains in doubt. ${ }^{6}$ This doubt is concerning because the therapeutic decision between ESD and surgery for EGC with a differentiated histology might primarily depend on the presence of the submucosal invasion of the tumor. If EUS does not decrease the proportion of additional treatments after ESD, or that of unnecessary surgery, then there is no need to perform EUS before EGC.

A recent study by Lee et al., ${ }^{7}$ published in Gut and Liver, has approached this issue directly. In that study, EUS did not increase the likelihood of selecting the appropriate treatment for
EGC with a differentiated histology. The appropriate treatment selection rates were $75.3 \%$ and $71.5 \%$ for the endoscopy- and EUS-based approaches, respectively, and these rates were not significantly different. In the case of endoscopically presumed mucosal cancer, however, an EUS-based treatment approach was inferior to its endoscopy-based counterpart. The authors demonstrated the nonrequirement for EUS by using a hypothetical treatment algorithm. Performing EUS routinely before treatment may not be necessary in patients with EGC.

However, certain issues remain to be discussed. All patients with EGC undergo endoscopic examination regardless of whether they undergo EUS examination. No endoscopist should ignore the endoscopic findings during selection of the treatment plan. Therefore, therapeutic decision-making on the basis of EUS alone is unrealistic. Furthermore, a treatment plan based on endoscopy with EUS might be more clinically meaningful than a treatment plan based on EUS alone. Lee et al. ${ }^{7}$ have reported that seven of 45 patients (8.3\%) with endoscopically presumed submucosal cancer successfully underwent ESD after an EUSbased treatment plan. In contrast, only one patient (1.2\%) with endoscopically presumed submucosal cancer needed surgery after ESD after an EUS-based treatment plan. Although this finding was not significant, it suggests that EUS may play a supporting role in conventional endoscopy. Here, we suggest an algorithm for determining the depth of tumor invasion by using both endoscopy and EUS in patients with EGC with a differentiated histology (Fig. 1). Using this algorithm, EUS is necessary only when EGC is estimated to be beyond the absolute indica-

Correspondence to: Sang Kil Lee

Department of Internal Medicine, Institute of Gastroenterology, Yonsei University College of Medicine, 50-1 Yonsei-ro, Seodaemun-gu, Seoul 03722, Korea

Tel: +82-2-2228-1996, Fax: +82-2-393-6884, E-mail: sklee@yuhs.ac

pISSN 1976-2283 eISSN 2005-1212 http://dx.doi.org/10.5009/gnl15387

@) This is an Open Access article distributed under the terms of the Creative Commons Attribution Non-Commercial License (http://creativecommons.org/licenses/by-nc/4.0) which permits unrestricted non-commercial use, distribution, and reproduction in any medium, provided the original work is properly cited. 


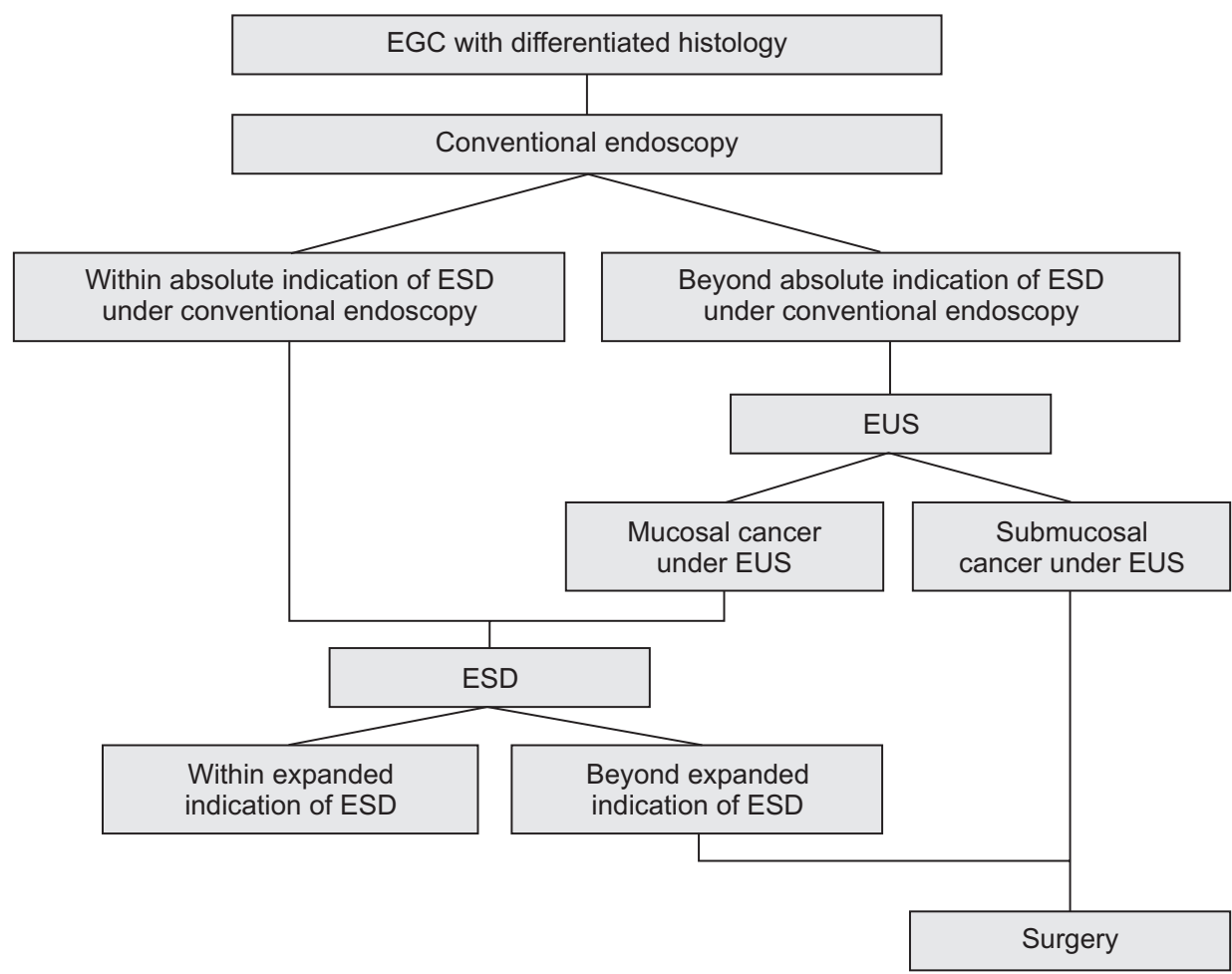

Fig. 1. An algorithm for making designing a treatment plan in for patients with early gastric cancer (EGC) with a differentiated histology. ESD, endoscopic submucosal dissection; EUS, endoscopic ultrasonography.

tion for ESD upon conventional endoscopy. When the tumor appears to be confined to the mucosa upon EUS, ESD is recommended as the initial treatment method even when the lesion appears to be beyond the absolute indication for ESD upon conventional endoscopy. This algorithm emphasizes the supporting role of EUS in minimizing the number of patients who undergo unnecessary surgery. We hope that the supporting role of EUS will be proven through future studies.

Another discussion point is the variation in the gastric cancer population. In Korea and Japan, early-stage gastric cancer, especially mucosal cancer, is more prevalent than advanced gastric cancer. ${ }^{1,8-10}$ Lee et al. ${ }^{7}$ have shown that the sensitivity for predicting mucosal cancer is higher for the diagnosis based on endoscopy (endoscopy, 83.2\% vs EUS, 66.9\%), whereas the specificity for predicting mucosal cancer is higher for the diagnosis based on EUS (endoscopy, 56.5\% vs EUS, 86.8\%). Given that the overall accuracy of a diagnostic tool with a high sensitivity and low specificity increases as the incidence or prevalence increases, an endoscopy-based treatment plan should be favored over an EUS-based treatment plan in Korea and Japan. However, in Western countries where mucosal cancer is less prevalent, EUS might play a larger role in planning the treatment strategy. If mucosal cancer is extremely rare among EGCs, then the relatively low specificity of endoscopy might significantly increase the performance of unnecessary ESD procedures under a treatment plan based on endoscopy alone.

Lee et al. ${ }^{7}$ have described the clinical role that EUS plays in choosing between ESD and surgery for patients with EGC. Routine EUS examination for therapeutic decision making may no longer be necessary. However, EUS might be an optional diagnostic modality because of its discriminatory ability in endoscopically presumed submucosal cancer. In addition, the relatively higher specificity of EUS compared with endoscopy should be considered when discussing the accuracy of EUS for predicting mucosal cancer because this accuracy depends on the incidence of mucosal cancer.

\section{CONFLICTS OF INTEREST}

No potential conflict of interest relevant to this article was reported.

\section{REFERENCES}

1. Park CH, Shin S, Park JC, et al. Long-term outcome of early gastric cancer after endoscopic submucosal dissection: expanded indication is comparable to absolute indication. Dig Liver Dis 2013; 45:651-656.

2. Isomoto H, Shikuwa S, Yamaguchi N, et al. Endoscopic submucosal dissection for early gastric cancer: a large-scale feasibility study. Gut 2009;58:331-336.

3. Gotoda T. Endoscopic resection of early gastric cancer. Gastric Cancer 2007;10:1-11.

4. Puli SR, Batapati Krishna Reddy J, Bechtold ML, Antillon MR, Ibdah JA. How good is endoscopic ultrasound for TNM staging of gastric cancers? A meta-analysis and systematic review. World J Gastroenterol 2008;14:4011-4019.

5. Mocellin S, Marchet A, Nitti D. EUS for the staging of gastric can- 
cer: a meta-analysis. Gastrointest Endosc 2011;73:1122-1134.

6. Choi J, Kim SG, Im JP, Kim JS, Jung HC, Song IS. Is endoscopic ultrasonography indispensable in patients with early gastric cancer prior to endoscopic resection? Surg Endosc 2010;24:3177-3185.

7. Lee JY, Choi IJ, Kim CG, et al. Therapeutic decision-making using endoscopic ultrasonography in endoscopic treatment of early gastric cancer. Gut Liver 2016;10:42-50.

8. Park CH, Kim B, Chung H, et al. Endoscopic quality indicators for esophagogastroduodenoscopy in gastric cancer screening. Dig Dis
Sci 2015;60:38-46.

9. Gotoda T, Yanagisawa A, Sasako M, et al. Incidence of lymph node metastasis from early gastric cancer: estimation with a large number of cases at two large centers. Gastric Cancer 2000;3:219225.

10. Takatsu Y, Hiki N, Nunobe S, et al. Clinicopathological features of gastric cancer in young patients. Gastric Cancer. Epub 2015 Mar 10. http://dx.doi.org/10.1007/s10120-015-0484-1. 